Revue Française de Civilisation Britannique

\title{
La finance éthique et la crise de 2008 : l'exemple britannique
}

Ethical Finance and the crisis of 2008: the case of Britain

\section{Aymen Boughanmi}

\section{CpenEdition}

Journals

Édition électronique

URL : http://journals.openedition.org/rfcb/1038

DOI : $10.4000 /$ rfcb.1038

ISSN : 2429-4373

Éditeur

CRECIB - Centre de recherche et d'études en civilisation britannique

Référence électronique

Aymen Boughanmi, «La finance éthique et la crise de 2008 : l'exemple britannique », Revue Française de Civilisation Britannique [En ligne], XXI-2 | 2016, mis en ligne le 10 septembre 2016, consulté le 03 mai 2019. URL : http://journals.openedition.org/rfcb/1038; DOI : 10.4000/rfcb.1038

Ce document a été généré automatiquement le 3 mai 2019.

\section{c)}

Revue française de civilisation britannique est mis à disposition selon les termes de la licence Creative Commons Attribution - Pas d'Utilisation Commerciale - Pas de Modification 4.0 International. 


\title{
La finance éthique et la crise de 2008 : l'exemple britannique
}

\author{
Ethical Finance and the crisis of 2008: the case of Britain
}

Aymen Boughanmi

\section{Introduction}

1 La réaction hostile que la crise de 2008 a suscitée contre le secteur financier s'inscrit dans une ancienne tradition de rejet moral et social des pratiques spéculatives. La condamnation unanime des religions monothéistes de l'usure ${ }^{1}$, considérée comme l'instrument des riches pour profiter des pauvres, fournit une source séculaire d'inspiration pour tout discours normatif contre le pouvoir de l'argent ${ }^{2}$. Cependant, la crise a montré clairement l'enchevêtrement quasi organique de la finance dans l'économie réelle : l'effondrement de l'une causerait obligatoirement la détérioration de l'autre. Ainsi, les critiques acerbes contre la finance n'ont pas empêché les États les plus puissants de rappeler les deniers publics au chevet des institutions bancaires ${ }^{3}$.

2 La frustration qu'a générée une telle nécessité se trouve à l'origine d'une réflexion féconde sur la nature de la crise et sur les solutions structurelles qu'il faut y apporter. La finance, considérée par beaucoup de commentateurs comme l'origine du mal, ne pouvait guère échapper à un tel effort. L'idée d'une finance éthique a ainsi connu un essor inédit. Fidèle à sa longue histoire d'innovation financière, le Royaume-Uni a constitué un laboratoire d'idées pour l'élaboration des principes et le développement des pratiques de cette tendance.

3 Cet article se propose d'étudier quelques exemples relatifs au phénomène de la finance éthique afin d'apporter quelques éléments de réponses à la question suivante : comment la crise de 2008 a-t-elle contribué à l'évolution de ce concept ?

4 Certaines tentatives destinées à introduire une dimension morale dans les pratiques du secteur financier ont pris une tournure quasi idéologique; d'autres se sont proposées d'apporter des réponses pratiques aux défis révélés par la crise. Ainsi, cet article est 
constitué de deux parties: la première analysera très rapidement les enjeux terminologiques et idéologiques soulevés par l'expérience britannique dans la finance éthique, à travers notamment les exemples de la finance écologique et de la finance islamique ; la deuxième présentera certaines tentatives qui visent à faire de la finance un instrument d'intégration économique et sociale.

\section{Les enjeux de la finance éthique au Royaume-Uni}

\section{Enjeux terminologiques}

5 Il est nécessaire de préciser que, dans un sens, toute finance peut être considérée comme éthique. Dans ce sens, l'éthique renvoie à toute déontologie qui régule les pratiques professionnelles des différents métiers ${ }^{4}$. En adéquation avec les valeurs postmodernes, la diversité de l'éthique est en train de supplanter le principe de l'indivisibilité de la morale universelle. Par conséquent, tout secteur qui fonctionne selon des règles spécifiques est objectivement éthique, même s'il n'est pas nécessairement moral. La finance conventionnelle ne fait pas exception. L'interconnexion de ses institutions en fait même un secteur structurellement solidaire malgré son caractère foncièrement concurrentiel. Ainsi, l'usage des termes «finance éthique» ou «finance solidaire» n'est pas objectivement approprié. Ce manque de précision explique, au moins en partie, la multiplication de termes concurrents qui s'efforcent de décrire des pratiques bancaires ou financières qui, sans nécessairement renoncer à la valeur du profit, tentent de se conformer à un certain nombre d'exigences sociales, morales ou religieuses. On parle ainsi de finance alternative, d'investissement durable ou d'investissement socialement responsable ${ }^{5}$.

6 Cependant, dans cet article, on utilisera principalement le terme "finance éthique ». Indépendamment de sa valeur objective, ce terme traduit une volonté de différentiation qui ne se limite pas à la dimension commerciale. Or, la terminologie utilisée pour le marketing contribue activement à définir cette distinction, et en conséquence, à délimiter un nouveau champ de concurrence entre les différentes composantes de ce marché. Puisque l'objectif est de faire dialoguer les valeurs fondamentales qui sont la raison d'être de ces composantes, le contenu commercial que traduit cette terminologie rend plus aisée la tâche de montrer leurs points de convergence et de divergence.

7 Concernant ce contenu, les Nations-Unies ont établi des principes pour un investissement responsable sur le plan social, environnemental et de la gouvernance. Cependant, ces principes sont relativement vagues. En outre, ils appellent à une approche inclusive des différentes entreprises, approche que la finance qui se dit «éthique " peut difficilement accepter sans risquer de perdre sa spécificité. Les mécanismes de l'offre et de la demande doivent être l'instrument principal d'un changement positif fondé sur l'évolution des choix des individus. Ainsi, il s'agit surtout de sensibiliser le public sans tomber dans l'activisme, parfois radical, que prônent les défenseurs d'un engagement déterminé en faveur de causes spécifiques. Selon ce modèle, l'Association Britannique pour un Investissement et une Finance Durables se définit comme une organisation pour la promotion d'un secteur financier socialement responsable. Les institutions bancaires qui la composent couvrent aussi bien les institutions traditionnelles que des banques ouvertement engagées pour des causes sociales, environnementales ou religieuses. Malgré cette diversité, elle persiste à justifier son existence par la défense des valeurs 
morales ${ }^{6}$. Toutefois, sa nature inclusive l'oblige à une modération dans l'approche et à une prudence dans l'action. Nécessairement limité, son objectif semble davantage être de promouvoir les bonnes pratiques que de dénoncer les mauvaises. Une telle attitude ne correspondait pas à l'atmosphère qui a suivi la crise de 2008 - crise qui a préparé le terrain pour une forme plus radicale d'activisme aspirant à une finance véritablement éthique.

8 En effet, au moment où la crise battait son plein, le discours officiel aux États-Unis et en Europe a clairement exprimé une profonde impuissance face au capital financier qui donnait l'impression de prendre en otage la volonté des pouvoirs publics, et qui menaçait, par conséquent, d'enrayer toute délibération démocratique le concernant. Cette tendance ploutocratique a créé une forte demande économique pour le développement de services financiers alternatifs qui intègrent certaines valeurs morales dans leurs politiques commerciales ${ }^{7}$.

9 Face à cette demande, le marché a répondu favorablement en proposant des solutions fondées sur des valeurs normatives diverses, telles que la mutualisation des risques, la durabilité environnementale, la responsabilité sociale ou les convictions religieuses. Même si la plupart des produits proposés existaient au Royaume-Uni bien avant 2008, il est important de noter que les circonstances de la crise ont fourni un arrière-plan très favorable pour une plus grande réceptivité de leur discours publicitaire. C'était la période où "l'éthique » s'est imposée comme la panacée de notre époque. En exigeant une plus grande conformité de la part du secteur financier avec ce principe, le discours officiel a implicitement reconnu le bien-fondé de la finance éthique qui se définit par opposition au secteur conventionnels.

10 Cependant, le mouvement pour une finance éthique souffre manifestement de l'ambiguïté fondamentale inhérente à la normativité postmoderne de la consommation responsable. En effet, la société de consommation, comme toute autre société, produit des valeurs qui permettent aux individus de conjuguer les avantages de ses pratiques avec la conviction d'une certaine supériorité morale'. En l'occurrence, l'une des difficultés consiste à établir une définition consensuelle du contenu que cache le label publicitaire de la finance éthique. En l'absence d'un tel contenu, ce mouvement risque de perdre son attrait, et de devenir, au mieux un rideau de fumée idéologique ${ }^{10}$, au pire un instrument de manipulation pour capter une clientèle réfractaire ${ }^{11}$. Mais, d'un autre côté, la rigueur conceptuelle qu'un tel contenu exige risque d'encombrer l'action d'institutions exposées à la concurrence d'une finance traditionnelle extrêmement dynamique et manifestement déterminée à rejeter tout reniement de la dérégulation acquise depuis l'époque thatchérienne.

11 Pour avoir une idée de l'ambiguïté des concepts constitutifs de la finance éthique, il suffit de s'interroger sur ce qui est censé être durable dans le cas de l'investissement : s'agit-il de l'investissement lui-même, par opposition à la spéculation à court terme ; ou de l'objet de l'investissement, qui doit ainsi donner la priorité à la protection de l'environnement ; ou encore, de l'effet social de l'investissement en termes de création et de redistribution de richesse ? Même si ces dimensions se sont croisées à l'occasion de la crise de 2008, il n'en demeure pas moins que leurs contradictions sont parfois fondamentales. Par exemple, la prudence excessive des mouvements écologiques est souvent perçue comme une entrave idéologique défavorable au climat des affaires. La plupart des investisseurs qui se définissent comme " éthiques » refusent d'établir une synonymie exclusive entre ce qui est acceptable du point de vue de la morale d'une part, et ce qui est durable sur le 
plan environnemental d'autre part. De la même façon, les contraintes auxquelles est soumise la finance islamique peuvent être considérées comme trop restrictives pour un secteur aussi dynamique que la finance, mais trop libérales pour un véritable engagement en faveur de la protection environnementale ${ }^{12}$.

Ainsi, l'impression d'unité que laisse entendre l'accord sur l'épithète "éthique " ne correspond que partiellement à la réalité. Certes, ces défis ne sont pas le produit de la crise financière de 2008. Leur origine se trouve dans la rupture conceptuelle provoquée par l'idée de l'engagement éthique d'un secteur conçu pour être moralement neutre ${ }^{13}$. Cependant, la crise a suscité une grande suspicion à l'égard de la finance, et a accru, en conséquence, l'exigence de clarté et de transparence vis-à-vis de toute institution qui se dit différente au sein de ce secteur. Perçue comme un refuge pour des consommateurs engagés, la finance éthique ne peut durablement maintenir son image positive qu'en démontrant qu'elle n'est pas une simple marque commerciale au service des institutions bancaires.

Le danger est d'autant plus réel qu'il est toujours plus facile de développer une conception négative fondée sur ce que la finance éthique ne peut pas inclure, comme la spéculation excessive, ou sur les activités à éviter, comme le secteur de l'armement. Une telle approche donnerait l'impression qu'il s'agit moins de développer l'engagement éthique de la finance que d'adapter les anciennes pratiques à une réalité commerciale imposée par la crise. En d'autres termes, la finance éthique risque de subir un effet boomerang si ses institutions se contentent d'une approche publicitaire implicitement comparative dépeignant la finance conventionnelle comme non-éthique.

L'absence de normes universelles qui déterminent la politique de sélection rend difficile la promotion de leurs choix économiques. La diversité de ces choix et la confusion des arguments qui les justifient risquent de mettre en péril la réputation que les institutions financières qui se définissent comme éthiques ont acquise pendant la crise. En effet, la crédibilité même de leur parole publique se trouve fragilisée par leur propre logique commerciale. Car il est évident qu'il n'est pas possible d'envisager un investissement fondé exclusivement sur des raisons éthiques, sans prendre en considération sa rentabilité économique. Un tel investissement peut mettre en péril la cause même qu'il prétend défendre, puisque son échec inéluctable exclut toute possibilité de le transformer en un choix économiquement viable.

La consolidation de la finance éthique nécessite donc un succès commercial durable. Or, dans le cadre d'une approche négative de cette finance, toute réussite encourt le risque d'être accusée d'utiliser les valeurs des individus pour des raisons non-éthiques. Le soupçon de manipulation est d'autant plus simple à établir qu'il est structurellement impossible de séparer les fins et les moyens. Comment faire la distinction entre un capital qui investit dans l'agriculture pour contribuer au développement de ce secteur, et celui qui fait le même choix économique par spéculation sur l'augmentation des prix de la terre et des produits agricoles?

\section{Risque d'idéologisation}

La crise économique de 2008 a fortement accru le besoin de politiques volontaristes qui se donnent pour objectif d'améliorer les choses plutôt que de se contenter d'un positionnement négatif qui évite certains secteurs économiques. En effet, la gravité de ses conséquences a révélé au public les défaillances des dispositifs de management et de 
contrôle des systèmes financiers. La transparence devient ainsi l'objet d'un large consensus qui unit les investisseurs, les actionnaires et le public. L'enjeu de la gouvernance occupe aujourd'hui une place centrale dans le débat sur la consolidation du secteur financier en général, et des institutions qui se veulent éthiques en particulier.

Un tel débat offre à la finance éthique une aubaine pour adopter une approche plus positive qui explique à la fois les valeurs à promouvoir, et la contribution de la finance engagée dans leur concrétisation. À titre d'exemple, la banque néerlandaise Triodos, dont la filiale britannique est située à Bristol, se définit comme une institution bancaire qui s'efforce de limiter ses financements aux activités favorables à l'environnement et à la société, et ne se contente donc pas d'éviter les secteurs négatifs. En septembre 2012, le fonds d'investissement Triodos Fund a été lancé au Royaume-Uni avec pour objectif d'investir dans les entreprises qui œuvrent pour la protection de la planète ou qui proposent une production durable ${ }^{14}$. Destiné à une clientèle déjà convaincue, un tel volontarisme réduit fortement le besoin d'activités publicitaires du groupe qui utilise principalement internet comme moyen de communication ${ }^{15}$. Mais une telle approche néglige clairement des valeurs comme l'inclusion des individus qui n'ont pas accès aux services bancaires traditionnels. Pour un écologiste qui ne définit pas son engagement éthique à partir d'une conception exclusivement environnementale, l'approche adoptée par la Banque Triodos peut paraître excessivement réductrice.

Demeure, cependant, un risque de taille puisque le volontarisme résidant dans toute conception positive de la finance éthique menace de la transformer en une idéologie économique. Or, il convient de rappeler que c'est d'abord une pratique commerciale et que la rentabilité économique demeure au cœur des préoccupations des institutions bancaires, même quand elles se vantent de leur engagement moral. Leur objectif est généralement de proposer des produits qui assurent une conciliation entre équité et rentabilité. C'est ainsi que l'entrepreneuriat financier espère échapper aux clichés que la crise de 2008 n'a fait que renforcer. Par exemple, dans son rapport annuel de 2010, la Banque Triodos a critiqué l'instabilité des incitations fiscales en faveur des énergies renouvelables qui constituent l'une des destinations majeures de ses investissements ${ }^{16}$. De tels commentaires montrent que, nonobstant les intentions initiales, l'hostilité vis-à-vis de la finance traditionnelle a bel et bien créé un effet d'aubaine en faveur d'une autre finance. En d'autres termes, la finance éthique peut être accusée de constituer une tentative pour faire coïncider le contexte économique et social de l'après-crise avec des doctrines préexistantes.

Dans cette perspective, d'aucuns estiment que la priorité consiste à déterminer les dérives et à mettre en place les politiques qui permettent de les éviter. Une telle attitude est nettement moins exclusive que les approches positives fondées sur des engagements à caractère idéologique. En outre, l'usage publicitaire des valeurs morales crée nécessairement une ambiguïté sur l'ordre des priorités. Idéalement, la finance éthique devrait être un pacte entre un investisseur engagé et un client engagé, avec un partage mutuel des risques relatifs à leur engagement commun. Or, il est possible d'interpréter cette relation sous un angle plus pragmatique: il s'agirait d'abord d'un engagement contractuel classique de la part d'une institution bancaire qui, en contrepartie d'un profit, fournit à sa clientèle la possibilité d'investir dans un type spécifique d'activités économiques. Dans ce sens, l'aspect éthique dérive essentiellement des choix des clients.

Ainsi, les succès de la finance dite « éthique » constituent une nouvelle démonstration de l'extraordinaire capacité de l'idéologie libérale à s'adapter aux différentes circonstances. 
L'hostilité du public vis-à-vis du secteur financier a généré une demande que le marché s'efforce de satisfaire. C'est ainsi que des banques traditionnelles au Royaume-Uni offrent, de plus en plus, des services conformes aux règles de la finance islamique ${ }^{17}$. Pour les clients idéologiquement désireux de se conformer à la doctrine orthodoxe de la charia, une telle solution pose le problème du manque d'insularité vis-à-vis des pratiques traditionnelles considérées comme haram ${ }^{18}$. En effet, c'est le respect des deux principes fondamentaux du halal (le permis) et du haram (l'interdit) qui donne une dimension éthique à la finance islamique. Ainsi, contrairement aux autres formes de finance éthique, la finance islamique se distingue par son détachement idéologique des circonstances économiques conjoncturelles. Instituée selon des règles préétablies et soumise au contrôle d'experts spécialisés, elle peut difficilement être accusée de se conformer à la simple évolution des goûts passagers des consommateurs ${ }^{19}$.

21 Après 2008, les institutions bancaires dites islamiques ont souvent fait preuve d'une solidité relative qui leur a permis de valoriser leurs spécificités. Il serait utile de rappeler que les principales interdictions que la finance islamique s'efforce d'éviter sont, premièrement, le gain garanti par le biais du riba (usure) ${ }^{20}$, et deuxièmement, les activités spéculatives collectivement nommées gharar (incertitude) ${ }^{21}$. En conséquence, la finance islamique fonde le contenu de son engagement moral sur le partage des risques et sur l'ancrage dans l'économie réelle ${ }^{22}$. Les circonstances exceptionnelles de la crise de 2008 ont été particulièrement favorables à la valorisation du modèle économique qui découle de ses principes. Fondé sur un partenariat entre l'investisseur et l'entrepreneur, ce modèle fait l'objet d'une attention croissante, même si des interrogations demeurent sur la capacité des institutions bancaires islamiques à hisser leurs pratiques au niveau de cet idéal ${ }^{23}$.

22 Cependant, l'affiliation religieuse de la finance islamique donne une dimension exclusive contraire aux attentes du public. En se soumettant aux textes fondamentaux de l'islam, mais surtout à l'interprétation qu'attribuent les experts religieux à ces textes, ce type de finance ne répond pas nécessairement aux urgences économiques et sociales de l'époque. Même sa stabilité morale peut facilement être compromise par l'évolution, souvent rigoriste, des différentes interprétations des textes. Son isolement signifie sa dépendance fondamentale de la popularité de l'engagement religieux au sein de la communauté musulmane. Ainsi, le capital devient l'allier de la ferveur religieuse qui motive sa clientèle. Loin de révolutionner la finance, cette offre se limite à l'adaptation des pratiques financières à des règles purement formelles et considérées comme obligatoires pour des raisons exogènes.

$\mathrm{Au}$ Royaume-Uni la Banque Islamique Britannique ${ }^{24}$ s'adresse essentiellement à la communauté musulmane et aux investisseurs internationaux désireux de faire fructifier leurs capitaux dans le respect de la charia. En outre, la conviction selon laquelle son encrage religieux lui procure une valeur morale incontestable l'isole au sein même de la finance éthique. À l'instar de la plupart des banques islamiques, elle semble davantage préoccupée par la conformité formelle de ses transactions avec les préceptes de l'Islam que par la compatibilité de ses pratiques avec celles des autres institutions de la finance éthique. L'importance des pétrodollars dans ses souscriptions la place en porte-à-faux avec l'engagement écologique, même si la plupart des investisseurs de cette tendance ne sont pas aussi catégoriques que la Banque Triodos.

24 Cet exemple montre l'ampleur de la difficulté d'évoluer vers une cohérence dans les différentes conceptions de la finance éthique. Les incompatibilités idéologiques rendent 
très difficiles toute coopération constructive entre les différentes composantes de ce secteur. Une modération dans les différentes approches serait la seule solution envisageable. C'est ce que tente de réaliser le Stewardship Fund ${ }^{25}$. Tout en reconnaissant la nécessité d'un engagement actif pour faire face à l'enjeu du changement climatique, ce fonds d'investissement, qui se veut éthique, ne s'interdit pas, par exemple, toute collaboration avec le secteur des énergies fossiles. Plutôt pragmatique, son approche consiste à utiliser l'investissement comme levier pour encourager les entreprises à limiter les effets négatifs de leurs activités économiques ${ }^{26}$.

Cette politique est de nature à déplaire à certains écologistes qui considèrent que les fonds disponibles devraient, en priorité, financer l'énergie du futur ou l'économie sociale et solidaire ${ }^{27}$. Poussé à son extrême, ce discours implique le refus de tout investissement dans les actions de grandes compagnies. Même le Triodos Fund rejette une telle approche, puisqu'elle signifie une rupture totale entre sa conception écologiste de la finance éthique d'une part, et la majorité des activités économiques d'une autre part. Cette réalité économique se traduit dans le discours commercial par la nécessité d'encourager les entreprises à poursuivre leurs efforts pour se conformer aux principes de l'économie durable. En outre, il est rappelé que toute évolution positive dans les pratiques environnementales des grandes entreprises peut avoir un effet plus important que la pureté de certaines petites structures ${ }^{28}$.

Ces exemples montrent la difficulté que pose la normalisation institutionnelle des approches dites éthiques de la finance. Malgré les possibilités de convergence que l'engagement moral de la finance peut promettre, la mise en place de politiques effectives pour le promouvoir génère souvent des contradictions insurmontables à trois niveaux : le développement d'approches cohérentes sur la relation entre principes idéologiques et réalité économique; la nécessité de répondre aux urgences sans tomber dans l'opportunisme commercial ; la coopération entre les différentes branches de la finance éthique. C'est peut-être donc sur le terrain qu'il serait possible d'apprécier la valeur réelle de cet engagement.

\section{Pour un endettement éthique}

\section{L'exemple des sociétés de crédit immobilier}

Compte tenu des conséquences de la crise économique, l'inclusion des catégories sociales les plus défavorisées, y compris dans le secteur bancaire et financier, constitue désormais un critère fondamental pour évaluer l'engagement éthique de toute institution bancaire ou financière. Cependant, ce consensus revêt une contradiction fondamentale qui traduit un aveu d'impuissance face à la mutation financière du capitalisme depuis les années 1980. En effet, c'est l'intégration d'un nombre de plus en plus important de consommateurs des services de crédit qui se trouve à l'origine de l'augmentation réelle de la dette dont le caractère systémique a préparé le terrain pour la crise. La dérégulation du secteur financier a progressivement placé la propriété immobilière au cœur de la notion de richesse. Si l'on définit la richesse comme l'actualisation de l'ensemble des revenus, on peut même parler d'un renversement de relation entre richesse et revenu.

Ainsi, malgré l'accélération de la consommation, la richesse pouvait augmenter sans aucune évolution adéquate des revenus. Par conséquent, le ratio dette sur richesse baissait au moment où le ratio dette sur revenu augmentait ${ }^{29}$. Le système financier 
fonctionnait comme si la valeur des actifs immobiliers devait suivre une courbe infinie à la hausse. Puisque ces actifs constituent le collatéral classique de l'endettement, et puisque les acheteurs et les vendeurs tablaient sur des calculs similaires, l'offre et la demande de crédit se sont nourries mutuellement ${ }^{30}$. En d'autres termes, grâce à la constance de l'augmentation des prix de l'immobilier, les individus ont progressivement ajusté leur comportement à l'idée de la disponibilité permanente des crédits ${ }^{31}$.

La crise a révélé l'erreur de ce type de raisonnement. La dépendance excessive de l'endettement vis-à-vis du collatéral immobilier a rendu fondamentalement instable la capacité de paiement des individus. Ainsi, la source de richesse est devenue une source de pauvreté $^{32}$. Cette crise immobilière constitue un défi éthique de taille, car elle concerne un besoin de première nécessité : le logement. Dans ce contexte, le modèle économique des sociétés de crédit immobilier a réussi à se distinguer. Fondé sur le principe de la mutualisation, il a structurellement protégé ces institutions des excès de la dérégulation. Le Building Societies Act de 1986 leur impose, par exemple, des restrictions sur l'accès aux marchés financiers et sur le recours aux produits financiers à risque ${ }^{33}$. Il est nécessaire de préciser qu'au Royaume-Uni, ce modèle ne date pas de 2008.

En effet, les sociétés de crédit immobilier sont le résultat d'une tradition mutualiste qui remonte au milieu du XIX ${ }^{\mathrm{e}}$ siècle. Depuis cette époque, des milliers de structures associatives s'étaient développées pour répondre aux besoins d'acquérir un bien immobilier pour les travailleurs. La dérégulation des années 1980 a radicalement changé la donne en permettant aux banques de concurrencer les sociétés de crédit immobilier sur leur terrain traditionnel. Allant croissant, leur capacité d'emprunter sur les marchés financiers a mis fin à la position monopolistique dont jouissaient jadis ces sociétés. Face à cette évolution, plusieurs de ces dernières ont choisi de se transformer en banques pour pouvoir défendre leur pré carré ou s'étendre vers de nouveaux horizons ${ }^{34}$.

31 Cette double tendance a conduit les structures bancaires à dominer progressivement l'emprunt immobilier. Mais les années de crise ont permis aux sociétés de crédit immobilier de renverser relativement la courbe en augmentant leurs parts de marché. En 2012, leur contribution a avoisiné $25 \%$ des crédits immobiliers au Royaume-Uni ${ }^{35}$. En outre, leurs expériences des restrictions imposées par la loi de 1986 dont elles bénéficient donnent une longueur d'avance sur leurs rivales qui tendent à rejeter instinctivement toute forme de régulation.

32 La crise a également été l'occasion de consolider les structures institutionnelles des sociétés de crédit immobilier. Cette consolidation s'inscrit dans une tendance lourde qui conduit inexorablement à dépasser le niveau local et régional des activités mutualistes. Après 2008, les plus petites structures locales avaient des difficultés à résister à la pression conjoncturelle. Elles étaient donc plus disposées à fusionner ou à être absorbées par les plus grandes sociétés. C'était ainsi que la Yorkshire Building Society a absorbé Barnsley, la Norwich, et la Chelsea. Cette tendance a permis l'émergence de sociétés de crédit immobilier qui, malgré leurs spécificités économiques, étaient en mesure de rivaliser avec les banques traditionnelles ${ }^{36}$.

з3 Forte de son histoire mutualiste et de sa taille imposante, la Yorkshire Building Society a choisi d'éviter les contradictions inhérentes à l'usage publicitaire des valeurs morales. Ainsi, elle évite de se présenter comme une institution éthique, bien que certaines de ses pratiques lui permettent largement de prétendre à ce titre. Par exemple, à travers son programme Small Change, Big Difference, elle encourage ses clients à renoncer à quelques 
pennies à chaque versement d'intérêt sur leurs comptes d'épargne, et de constituer ainsi des fonds qui sont versés à des projets sociaux. Mais l'essentiel de l'effort publicitaire de la Yorkshire Building Society se concentre sur le coût et la qualité de ses services. Tout en prenant le risque de perdre une part de la clientèle idéologiquement motivée, cette politique répond plus largement aux priorités du client moyen.

D'autres stratégies de communication peuvent être observées. Outre leur engagement mutualiste, certaines sociétés de crédit immobilier ont choisi d'orienter leurs efforts pour défendre activement des causes spécifiques, et de le faire savoir même à travers leurs raisons sociales. Comme son nom l'indique, l'Ecology Building Society œuvre, depuis sa création en 1981, pour la défense de l'environnement à travers la promotion des projets durables. Tous les services d'épargne qu'elle propose, comme l'Earthsaver Bond et l'Eco-60 Account, évoquent d'emblée cet enjeu. Aujourd'hui, sa publicité a pris une tournure franchement militante et passablement agressive puisqu'elle se fonde sur une comparaison explicite entre ses pratiques éthiques et les dérives de la finance traditionnelle. Après le scandale du LIBOR ${ }^{37}$, elle n'a pas hésité à stigmatiser les pratiques des institutions financières qui ne prennent en considération que les aspects comptables du gain matériel ${ }^{38}$.

Un tel discours vise à mettre davantage en valeur son propre engagement environnemental et social, et à réaffirmer que ces deux dimensions sont foncièrement indissociables. Sur ce chapitre, l'Ecology Building Society a su assurer une différenciation positive, non seulement au sein du marché éthique en général, mais également sur le segment écologique de ce marché. En combinant une approche radicale orientée vers une clientèle convaincue et engagée d'une part, et une démarche inclusive qui profite aux catégories les moins bien loties d'une autre, elle a réussi à dépasser la plupart des contradictions liées à la finance éthique. Une des mesures-phares de cette politique a été l'offre d'une baisse des taux d'intérêt conditionnée par l'efficacité énergétique des maisons construites ou rénovées. En orientant ainsi une partie des investissements vers des petits projets individuels, cette option assure l'inclusion de ceux qui ne pouvaient pas bénéficier d'initiatives plus globales. En acceptant de renoncer à une part de ses bénéfices, l'Ecology Building Society promeut l'idéal d'une finance éthique fondée sur un pacte entre un investisseur et un client qui œuvrent collectivement dans un cadre économique novateur pour la défense de causes communes. Le contrat qui réunit ces deux parties assure la rationalisation de leur engagement éthique sans rendre déséquilibrée la dimension émotionnelle qu'implique ce pacte.

\section{La dette entre exclusion et inclusion sociale}

En 2012, le gouvernement britannique a annoncé la création de la Big Society Capital. Il s'agit d'une structure bancaire dédiée au financement des entreprises qui œuvrent pour la promotion des valeurs sociales ${ }^{39}$. Cette initiative s'inscrit dans une logique globale adoptée par le gouvernement de coalition de l'époque entre Conservateurs et libéraux afin de réduire les responsabilités économiques et sociales de l'État ${ }^{40}$. Le secteur privé est encouragé à s'engager activement en faveur d'un investissement social durable, lui permettant de remplacer progressivement l'intervention publique pour assurer certains services à la communauté. Cette approche se fonde sur la conviction selon laquelle l'entrepreneuriat reste le moteur principal de tout changement. Par conséquent, il est 
crucial d'orienter son énergie vers le bien collectif; d'où l'idée d'une banque qui incarne l'expansion du principe de l'entreprise sociale au secteur de la finance.

Néanmoins, une telle politique est de nature à susciter la suspicion des défenseurs de l'État providence, qui sont souvent ceux-là mêmes qui appellent à une finance plus éthique. Cependant, le lancement de la Big Society Capital peut aussi être interprété comme l'aboutissement national d'un effort collectif pour réduire les effets indésirables de l'endettement et pour essayer de l'utiliser comme instrument d'intégration sociale. La question est la suivante: est-il possible de se servir de la dette comme un moyen d'intégration sociale, ou au moins comme instrument pour lutter contre l'exclusion? En d'autres termes, est-il possible de faire bénéficier les catégories les plus défavorisées des avantages de l'endettement, tout en minimisant les risques inhérents à la fragilité de leurs situations économiques et sociales?

La Big Society Capital soutient un fonds d'investissement social appelé Investing for Good. Ce fonds se spécialise dans l'adaptation du système de l'emprunt obligataire au profit des associations. L'objectif étant d'orienter une partie des investissements disponibles vers le secteur social, ces associations peuvent désormais financer leurs projets en émettant des titres avec une valeur nominale, des coupons et une maturité. Grâce à cette initiative, les investisseurs sont encouragés à soutenir les causes qui leur tiennent à cœur tout en réalisant un profit.

Parallèlement, dans le secteur bancaire, la Charity Bank représente un exemple particulièrement intéressant. Il s'agit d'une structure hybride enregistrée comme association et reconnue comme banque. Sans évoquer une relation asymétrique fondée sur le don aux pauvres par les riches, elle s'efforce d'adapter ses activités aux principes de l'aide à la personne et des services à la communauté. Outre les coûts très avantageux de ses services, la Charity Bank met en avant la stabilité de ses taux d'intérêt qui ne dépendent pas des variations des taux de la Banque d'Angleterre. Tout en suivant le modèle basique d'une banque, elle ne prête que sur projet à caractère social. Son objectif déclaré est de fournir une opportunité pour un investissement fructueux qui favorise la cohésion $^{41}$. Sa clientèle est donc essentiellement constituée de petites associations et de groupes de volontaires avec un ancrage solide au niveau local. Ses structures ont typiquement des objectifs charitables, tels que l'aide aux personnes vulnérables ou la promotion de l'éducation.

D'autres expériences se sont développées pour répondre à d'autres types de besoins. Par exemple, la pauvreté et la précarité professionnelle provoquent des situations d'urgence qui engendrent souvent une nécessité d'endettement. Or, c'est précisément dans ce genre de conditions qu'il est extrêmement difficile de négocier des termes favorables pour l'obtention d'un prêt. Les individus acceptent donc des taux usuraires, ce qui conduit à perdurer la situation d'urgence. À partir de la conclusion selon laquelle c'est l'absence d'emprunt abordable qui conduit au cercle vicieux de l'endettement, la Fair Finance a été créée pour lutter contre l'exclusion financière en répondant aux besoins d'endettement des personnes qui ne remplissent pas les conditions des crédits personnels classiques. Elle peut accorder des prêts allant jusqu'à 2000 livres à des taux d'intérêt qui permettent d'assurer la viabilité de l'entreprise. Elle propose également des conseils individualisés pour la gestion de l'endettement de ses clients. Ceux-ci sont systématiquement incités à utiliser les crédits accordés pour rembourser leurs anciennes dettes. En effet, l'un des objectifs de la Fair Finance est de combattre les pratiques qui consistent à profiter des difficultés des individus pour leur imposer des taux d'intérêt prohibitifs. Selon Faisel 
Rahman, le fondateur de cette structure, cette bataille n'est pas menée contre les banques, mais contre les autres organismes de crédit à l'instar de Wonga ${ }^{42}$. Pour lui, le monopole des prédateurs sur l'emprunt aux pauvres ne peut être brisé que par le biais d'un engagement social très fort de la part des banques ${ }^{43}$.

\section{Conclusion}

41 Malgré sa violence, la crise financière de 2008 ne semble pas avoir provoqué une rupture dans la conception générale des principes économiques ${ }^{44}$, puisque l'initiative privée et la libre entreprise demeurent le moteur de toute évolution espérée. La finance éthique peut être considérée comme une manifestation de cette constante. Aujourd'hui, compte tenu du recul forcé de l'État providence, c'est le secteur social qui fait l'objet de cette réflexion.

42 En revanche, la crise est peut-être en train de conduire à une rupture aussi radicale qu'inattendue dans la conception même de la générosité en tant que valeur morale. L'essor de la finance éthique signifie qu'une réflexion théorique et pratique est en marche. Contrairement aux apparences, cet effort ne se limite pas à reconsidérer le rôle de la finance dans la société. Il concerne également le sens de la cohésion sociale et les moyens nécessaires pour sa mise en œuvre. L'entrepreneuriat éthique supplante le don comme instrument de charité. D'aucuns peuvent bien sûr considérer qu'il s'agit là d'une nouvelle mutation du libéralisme, le capital cherchant à profiter du recul de l'Étatprovidence. Mais d'autres se félicitent d'ores et déjà d'une conception positive de l'aide sociale. Tout en préservant l'autonomie des individus, qui ne sont plus soumis à la manipulation émotionnelle liée à la charité traditionnelle, cette évolution offre à la société civile la possibilité d'échapper à l'emprise de l'état et aux calculs politiques des gouvernements.

43 Auteur de plusieurs livres et articles en français, en anglais et en arabe, Aymen Boughanmi enseigne l'histoire et la civilisation britannique à l'École Normale Supérieure de Tunis. Intitulée L'impérialisme britannique du libre-échange, 1846-1932: Une analyse géoéconomique, sa thèse propose une redéfinition de l'équilibre entre les enjeux stratégiques et économiques dans la détermination des priorités dans l'espace public.

\section{BIBLIOGRAPHIE}

AINLEY Michael et al., Financial Services Authority, Islamic Finance in the UK : Regulations and Challenges , (2007), <http://www.fsa.gov.uk/pubs/other/islamic_finance.pdf>, [consulté le 23 février 2015]. BAUDRILLARD Jean, La société de consommation, ses mythes, ses structures, (Paris, Denoël, 1970). BLOND Phillip, " There is no wealth but life », in WILLIAMs Rowan et ELLIOTT Larry (dir.), Crisis and Recovery: Ethics, Economics and Justice, (Londres, Palgrave, 2010), pp. 77-99. 
BUSH Nathaniel I., MARTELli Peter F. et ROBERTS Karlene H., « Failures of High Reliability in Finance ", in SHRIVASTAVA Paul et STATLER Matt (dir.), Learning from the Global Financial Crisis: Creatively, Reliably, and Sustainably, (Stanford CA, Stanford Business Books, 2012), pp. 167-187.

CABLE Vince, The Storm, (Londres, Atlantic, 2009).CROW Karim Douglas, " Consuming Islam: Branding 'Wholesome' as Lifestyle Fetish ", Islamic Sciences, vol. 13, n 1, (été 2015), pp. 3-28. DUNKELBERG William C., « Open Secret : The Global Banking Conspiracy That Swindled Investors out of Billions », Business Economics, vol. 50, (avril 2015), pp. 109-110.

HAWSER Anita, «Standard Bearers », Global Finance, vol. 24, n 10, (novembre 2010), p. 25. HENRIQUES Irene, et SADORSKY Perry, « Oil prices and the stock prices of alternative energy companies ", Energy Economics, vol. 30, (2008), pp. 998-1010.

HM TREASURY, The Future of Building Societies, (juillet 2012), <www.hm-treasury.gov.uk>, [consulté le 18 mars 2015].

HOLTERHUES Eric, « Socially Conscious Investment », Triodos Newsletter: The Colour of Money, (automne 2012).

HOUSBY Elaine, Islamic and Ethical Finance in the United Kingdom, (Edinburgh, Edinburgh University Press, 2013).

HOUSBY Elaine, Islamic Financial Services in the United Kingdom, (Edinburgh, Edinburgh University Press, 2011).

KINGSMILL Baroness, « How Brits Get by on Less », Management Today, $1^{\mathrm{er}}$ octobre, 2012, p. 22.

LABATT Sonia, et WHITE Rodney R., Carbon finance : The financial implications of climate change, (Hoboken NJ, Wiley, 2007).

LLYOD Carol, « Minorities Are the Emerging Face of the Subprime Crisis », 13 avril 2007, SF Gate, < http://articles.sfgate.com/2007-04-13/entertainment/17238073_1_mortgage-rates-zerodownmortgage-american-dream>, [consulté le 09 mars 2015].

MASOOD Omar, Islamic Banking and Finance: Definitive Texts and Cases, (New York, Palgrave MacMillan, 2011).

MAUER Bill, « Engineering an Islamic Future: Speculations of Islamic Financial Alternatives », Anthropology Today, vol. 17, $\mathrm{n}^{\circ}$ 1, (2001), pp. 8-11.

MCGOUN Elton G. et ZIELONKA Piotr, « The Platonic Foundations of Finance and the Interpretation of Finance Models ", Journal of Behavioral Finance, vol. 7, n 1, (2006), pp. 43-57.

MITROFF Ian I. et ALPASLAN Can M., « Wrong assumptions and Risk Cultures: Deeper Causes of the Global Financial Crisis ", in SHRIVASTAVA Paul et STATLER Matt (dir.), Learning from the Global Financial Crisis: Creatively, Reliably, and Sustainably, (Stanford CA, Stanford Business Books, 2012), pp. 188-198.

NEWLANDS Chris, « Green Funds: An Ethical Dilemma », Financial Times, 2 décembre 2010, < http:// www.ft.com/cms/s/0/5b318cce-fd61-11df-b83c-00144feab49a.html>, [consulté le 09 mars 2015].

NIZAMI Shah M., «Islamic Finance: The United Kingdom's Drive to Become the Global Islamic Finance Hub and the United States' Irrational Indifference to Islamic Finance », Suffolk Transnational Law Review, vol. 34, $\mathrm{n}^{\circ}$ 1, (hiver 2011), pp. 219-254.

PURDY David, «Crisis and Regime Change in Britain », Soundings, $\mathrm{n}^{\circ}$ 57, (printemps 2014), pp. 95-105. 
RAHMAN Faisel, «Banks can help the financially excluded », The Guardian, 27 avril 2011, <http:// www.theguardian.com/society/2011/apr/27/banks-can-help-financially-excluded-model>, [consulté le 09 mars 2015].

REES Eifion et LEE Matilda, « Ecologist Guide to Ethical Investments », The Ecologist, 5 novembre 2010, <http://www.theecologist.org/green_green_living/ecologist_guides/673622/ ecologist_guide_to_ethical_investments.html>, [consulté le 09 mars 2015].

ROBBINS Holly E., « Soul Searching and Profit Seeking: Reconciling the Competing Goals of Islamic Finance », Texas Law Review, vol. 88, n5, (2010), pp. 1125-1127.

SADORSKY Perry, « Green Financing After the Global Financial Crisis ", in SHRIVASTAVA Paul et STATLER Matt (dir.), Learning from the Global Financial Crisis: Creatively, Reliably, and Sustainably, (Stanford CA, Stanford Business Books, 2012), pp. 213-244.

SORKIN Andrew Ross, Too Big to Fail: The Inside Story of How Wall Street and Washington Fought to Save the Financial System-and Themselves, (New York, Penguin, 2009).

STARKIK Mark, «A Multilevel, Multisystems Strategic Approach to a Sustainable Economy », in SHRIVASTAVA Paul et STATLER Matt (dir.), Learning from the Global Financial Crisis: Creatively, Reliably, and Sustainably, (Stanford CA, Stanford Business Books, 2012), pp. 291-312.

TAWNEY Richard Henry, Religion and the Rise of Capitalism. A Historical Study, (Londres, John Murray, 1926).

THALER Richard, «From Homo economicus to Homo sapiens », Journal of Economic Perspectives, vol. $14, \mathrm{n}^{\circ} 1,(2000)$, pp. 133-141.

TRIODOS BANK, Annual Report, (2010), <https://www.triodos.com/downloads/about-triodos-bank/ annual-reports/annual-report-2010.pdf>, [consulté le 18 mars 2015].

WAUGH Jody et AL AWAMLEH Ahmad, « Recognition and Enforcement of Islamic Finance in the Middle East », Business Law International, vol. 15, n 1, (janvier 2014), pp. 35-44.

WEICK Karl E. et SUTCLIFFE Kathleen M., Managing the Unexpected: Assuring High Performance in an Age of Complexity, (San Francisco, Jossey-Bass, 2007).

WILson Rodney, Economics, Ethics and Religion: Jewish, Christian and Muslim Economic Thought, (Londres, Palgrave Macmillan, 1997).

\section{NOTES}

1. Voir WiLson Rodney, Economics, Ethics and Religion: Jewish, Christian and Muslim Economic Thought, (Londres, Palgrave Macmillan, 1997), p. 33.

2. TAWNEY Richard Henry, Religion and the Rise of Capitalism. A Historical Study, (Londres, John Murray, 1926), pp. 36-37.

3. Pour l'exemple américain, voir soRKIN Andrew Ross, Too Big to Fail: The Inside Story of How Wall Street and Washington Fought to Save the Financial System-and Themselves, (New York, Penguin, 2009).

4. Selon certaines interprétations postmodernes, les théories économiques et financières ne sont qu'une forme de convention sociale : la vérité n'est vérité que grâce à l'opinion positive que lui accordent les théoriciens et les praticiens. Ainsi, toute évaluation normative de la finance est nécessairement conditionnée par les pratiques du marché financier lui-même. Traduisant un relativisme éthique et épistémologique, cette vision réduit tout référentiel d'évaluation à un 
ensemble de règles de jeu collectivement perçues comme acceptables. Voir MCGOUN Elton G. et ZIELONKA Piotr, «The Platonic Foundations of Finance and the Interpretation of Finance Models ", Journal of Behavioral Finance, vol. 7, $\mathrm{n}^{\circ}$ 1, (2006), pp. 49-53.

5. Cette diversité traduit également le caractère multidimensionnel de la question. Voir STARIK Mark, «A Multilevel, Multisystems Strategic Approach to a Sustainable Economy», in SHRIVASTAVA Paul et STATLER Matt (dir.), Learning from the Global Financial Crisis: Creatively, Reliably, and Sustainably, (Stanford CA, Stanford Business Books, 2012), pp. 291-312.

6. UK Sustainable Investment and Finance Association (UKSIF). Voir HOUSBY Elaine, Islamic and Ethical Finance in the United Kingdom, (Edinburgh, Edinburgh University Press, 2013), pp. 46-48.

7. Pour l'exemple de la finance islamique, voir RoBBinS Holly E., «Soul Searching and Profit Seeking: Reconciling the Competing Goals of Islamic Finance », Texas Law Review, vol. 88, n5, (2010), pp. 1125-1127.

8. MITROFF Ian I. et ALPASLAN Can M., «Wrong assumptions and Risk Cultures: Deeper Causes of the Global Financial Crisis », in SHRIVASTAVA et STATLER (dir.), Learning, pp. 188-196.

9. Si l'on accepte l'idée de Baudrillard selon laquelle les individus ne fonctionnent plus comme citoyens, mais plutôt comme des consommateurs, un bon individu serait donc un consommateur responsable. BAUDRILLARD Jean, La société de consommation, ses mythes, ses structures, (Paris, Denoël, 1970).

10. Pour le cas de la finance islamique, voir MAUER Bill, «Engineering an Islamic Future: Speculations of Islamic Financial Alternatives », Anthropology Today, vol. 17, n 1, (2001), pp. 23-24. 11. Voir par exemple NEWLANDS Chris, "Green Funds: An Ethical Dilemma », Financial Times, (2 décembre 2010). [consulté le 09 mars 2015].

12. HENRIQUES Irene, et SADORSKY Perry, "Oil prices and the stock prices of alternative energy companies ", Energy Economics, vol. 30, (2008), pp. 998-1010 ; LABATT Sonia, et WHITE Rodney R., Carbon finance : The financial implications of climate change, (Hoboken NJ, Wiley, 2007).

13. THALER Richard, «From Homo economicus to Homo sapiens ", Journal of Economic Perspectives, vol. 14, n 1, (2000), pp. 133-141.

14. HOLTERHUES Eric, «Socially Conscious Investment ", Triodos Newsletter: The Colour of Money, (automne 2012).

15. HOUSBY, Islamic, (2013), p. 35.

16. TRIODOS BANK, Annual Report, (2010), p. 11.

17. En Juillet 2003, HSBC a lancé, au Royaume-Uni, une filiale islamique appelée Amanah UK. Deux années plus tard, Lloyds a également rejoint le marché islamique en proposant des produits conformes aux règles de la charia au sein de ses filiales situées dans des zones à forte présence musulmane. Plus généralement, depuis au moins trois décennies, le Royaume-Uni s'efforce d'intégrer la finance islamique dans le secteur bancaire traditionnel. Voir NIZAMI Shah M., « Islamic Finance: The United Kingdom's Drive to Become the Global Islamic Finance Hub and the United States' Irrational Indifference to Islamic Finance », Suffolk Transnational Law Review, vol. 34, $\mathrm{n}^{\circ} 1$, (hiver 2011), note 52 ; AINLEY Michael et al., Financial Services Authority, Islamic Finance in the UK: Regulations and Challenges, (2007), <http://www.fsa.gov.uk/pubs/other/ islamic_finance.pdf>, [consulté le 23 février 2015].

18. HOUSBY, Islamic, (2013), p. 21.

19. Il ne s'agit pas ici de suggérer que le label islamique est totalement étranger à la logique du marketing qui utilise les besoins psychologiques et spirituels des individus à des fins commerciales. Pour une analyse critique de la consommation dite islamique, voir, par exemple, CRow Karim Douglas, "Consuming Islam: Branding 'Wholesome' as Lifestyle Fetish », Islamic 
Sciences, vol. 13, n 1, (été 2015), pp. 3-28. Mais les critiques de la marchandisation de certaines croyances religieuses ne sont pas en contradiction avec l'idée selon laquelle les principes fondateurs de la finance islamiques sont intimement liés au caractère transcendantal de la charia en tant que loi révélée par Dieu.

20. L'opinion prédominante au sein des communautés musulmanes dit que tout paiement d'intérêt est généralement considéré comme riba. Cette vision néglige clairement l'argument qui soutient que c'est l'augmentation injustifiée de la valeur du capital qui se trouve à l'origine de l'interdiction de l'usure. Voir HOUSBY Elaine, Islamic Financial Services in the United Kingdom, (Edinburgh, Edinburgh University Press, 2011), pp. 17-19.

21. Dans ce contexte, il serait plus approprié d'utiliser le terme "spéculation». Voir ibid., pp. 14-15.

22. Pour un exemple d'instruments financiers dits islamiques, voir WAUGH Jody et $A L$ AWAMLEH Ahmad, « Recognition and Enforcement of Islamic Finance in the Middle East », Business Law International, vol. 15, $\mathrm{n}^{\circ} 1$, (janvier 2014), pp. 37-38.

23. HAWSER Anita, "Standard Bearers", Global Finance, vol. 24, n 10, (novembre 2010), p. 25 ; MASOOD Omar, Islamic Banking and Finance: Definitive Texts and Cases, (New York, Palgrave MacMillan, 2011), p. 209.

24. Lancée en 2004 par des capitaux du Golfe, la Banque Islamique Britannique est la première banque britannique qui limite ses activités aux services bancaires et aux produits financiers conformes à la charia. Tout en réduisant son potentiel de croissance, ce choix la rend particulièrement attractive aux Musulmans orthodoxes qui refusent tout contact avec la finance traditionnelle.

25. Lancé en 1984, le Stewardship Fund est le premier fonds d'investissement britannique qui se définit comme éthique. Ses investissements s'orientent vers des activités très différentes, mais leur sélection obéit à des critères communs qui peuvent être divisés en deux groupes: des critères positifs pour la promotion de certains investissements, et des critères négatifs destinés à l'exclusion d'autres activités.

26. Pour une étude des différents sénarios de ce type d'investissement, voir SADORSKY Perry, "Green Financing After the Global Financial Crisis », in SHRIVASTAVA et STATLER (dir.), Learning, pp. 291-312.

27. REES Eifion et LEE Matilda, « Ecologist Guide to Ethical Investments », The Ecologist, 5 novembre 2010.

28. HOLTERHUES Eric, Socially, (2012), p. 9.

29. Par exemple, une personne qui gagnait 15000 dollars par an a pu obtenir un crédit de 720000 dollars. Voir Lloyd Carol, "Minorities Are the Emerging Face of the Subprime Crisis ", 13 avril 2007, SF Gate, <http://articles.sfgate.com/2007-04-13/entertainment/17238073_1_mortgagerates-zerodown-mortgage-american-dream>, [consulté le 09 mars 2015].

30. Pour le caractère systémique de la crise, voir Nathaniel I. BUSH, Peter F. MARTELLI et Karlene H. ROBERTS, «Failures of High Reliability in Finance ", in Paul SHRIVASTAVA et Matt STATLER (dir.), Learning from the Global Financial Crisis: Creatively, Reliably, and Sustainably, pp. 167-186.

31. BLOND Phillip, « There is no wealth but life ", in WILLIAMS Rowan et ELLIOTT Larry (dir.), Crisis and Recovery: Ethics, Economics and Justice, (Londres, Palgrave, 2010), pp. 82-83.

32. Weick Karl E. et SUTCLIFFE Kathleen M., Managing the Unexpected: Assuring High Performance in an Age of Complexity, (San Francisco, Jossey-Bass, 2007), pp. 10-17.

33. HM TREASURY, The Future of Building Societies, (juillet 2012), <www.hm-treasury.gov.uk>, pp. 7-9, [consulté le 18 mars 2015]. 
34. Dans ce cadre, l'exemple de Northern Rock a acquis une notoriété spécifique après la crise de 2008. Pour plus de détails, voir CABLE Vince, The Storm, (Londres, Atlantic, 2009), pp. 9-14

35. HOUSBY, Islamic, (2011), p. 72.

36. HM TREASURY, The Future, (2012).

37. Le LIBOR est un acronyme pour London Interbank Offered Rate. Il s'agit d'un taux de prêts interbancaires que les marchés financiers utilisent comme référence pour évaluer les situations des banques. Avant 2008, pour dissimuler leurs problèmes de liquidités, les banques ont systématiquement manipulé ce taux. Par exemple, en déclarant un taux inférieur à la réalité, elles réduisaient les inquiétudes concernant leurs niveaux de liquidités. Pour un récit détaillé de ces manipulations, voir DUNKELBERG William C., «Open Secret: The Global Banking Conspiracy That Swindled Investors out of Billions », Business Economics, vol. 50, (avril 2015), pp. 109-110.

38. Communiqué de presse daté du 29 juin 2012, disponible sur : <www.ecology.co.uk>.

39. Big Society Capital, <www.bigsocietycapital.com>.

40. Il est nécessaire de préciser que cette logique s'inscrit dans la continuité de l'idée d'une troisième voie entre le socialisme et le libéralisme. Prôné par les gouvernements travaillistes depuis 1997, ce concept mettait déjà l'accent sur le rôle du secteur associatif et de l'entrepreneuriat privé dans la promotion de certains services à caractère social.

41. Charity Bank, <www.charitybank.org>.

42. KINGSMILl Baroness, « How Brits Get by on Less ", Management Today, $1^{\text {er }}$ octobre, 2012, p. 22.

43. RAHMAN Faisel, « Banks can help the financially excluded », The Guardian, 27 avril 2011.

44. PURDY David, «Crisis and Regime Change in Britain », Soundings, $n^{\circ} 57$, (printemps 2014), pp. 95-96.

\section{RÉSUMÉS}

Depuis 2008, la finance éthique est apparue comme une solution prometteuse pour combler le déficit moral que l'on reproche au secteur financier. Cependant, les espoirs que cette solution a fait naître posent plusieurs interrogations sur cette évolution particulière du système capitaliste et sur la nature de ses innovations idéologiques. Dans ce contexte, le Royaume-Uni représente un exemple significatif. Puisque son marché offre un cadre favorable pour des formes contradictoires de finance éthique, il semble utile d'étudier les différentes articulations de leurs principes fondamentaux, ainsi que leurs implications pratiques et idéologiques dans un contexte de crise économique.

Since 2008, ethical finance has emerged as a promising answer for the supposedly moral deficit of the financial sector. However, the hopes that such solution inspired raise many questions about this specific evolution of the capitalist system and the nature of its ideological innovations. In this context, United Kingdom represents a significant case. As its financial market offers a welcoming framework for conflicting forms of ethical finance, it seems important to study the different articulations of their founding principles, as well as their ideological and practical implications in a context of an economic crisis. 
INDEX

Mots-clés : finance éthique, crise économique, finance islamique, finance écologique, endettement

Keywords : ethical finance, economic crisis, Islamic finance, ecological finance, debt

\section{AUTEUR}

AYMEN BOUGHANMI

École Normale Supérieure de Tunis 